\title{
Spectrum Sensing in Cognitive Radio by Advanced Cuckoo Search
}

\author{
Ashu Malviya ${ }^{1}$, Swapnil Nema ${ }^{2}$ \\ Department of Electronics and \\ Communication \\ Global Nature Care Sangathan's Group
}

\author{
Of Institutions \\ Jabalpur, India \\ malviyaashu11@gmail.com ${ }^{1}$ \\ swapnil.nema27@gmail.com²
}

\begin{abstract}
The radio spectrum is a naturally limited resource which is needed for wireless communication systems. Cognitive Radio (CR) is a suitable technology that can improve the spectrum utilization efficiency. Spectrum utilization can be enhanced by allocating licensed band to secondary user $(\mathrm{SU}) /$ unlicensed user when the primary user (PU)/licensed user is absent. The key feature of the $C R$ is spectrum sensing. The aim of this paper is to sense the spectrum holes or channels and if primary user is not present then provide those empty channels to secondary users by using Advanced Cuckoo Search method (ACS). By using ACS method, the time of capturing free channels is reduced as compared to other methods. Furthermore, simulation is done to study the performance of proposed method, which shows the proposed scheme is more efficient as compared to existing schemes.
\end{abstract}

Keywords-Cognitive Radio, Spectrum Sensing, Cuckoo Search

\section{INTRODUCTION}

The radio frequency spectrum is one of the most valuable natural resource for wireless communication. Federal Communication Commission (FCC) report on spectrum utilization reveals that many licensed bands are not used for a large time duration and remains unoccupied. In highly populated area, the spectrum utilization hardly ever cross $35 \%$ at any given time [1]. Cognitive Radio (CR) is a key technology that provides efficient utilization of spectrum. Cognitive radio is an intelligent radio and network technology that detects available channels, in which secondary user can change particular transmission, reception, or radio environment sensing parameters to execute their tasks provided that no interference with licensed user. below:

The basic services of Cognitive Radio network are given

\section{Spectrum Sensing}

This is the ability of CR network to sense the unoccupied spectrum band. If Primary user is not active and channel is unoccupied then share it with Secondary user(Unlicensed user), under the condition that, primary user should not be disturbed..

\section{Spectrum Management}

The ability of CR network to find best spectrum bands to provide user requirements over all available spectrum bands.. Spectrum management can be divided into two functions .

a)spectrum analysis and

b)spectrum decision

\section{Spectrum Mobility}

In spectrum mobility, CR network changes its operating frequency and move to best available channel. Secondary user leaves the channel if the primary user comes into existence again and switch into another unoccupied channel.

\section{Spectrum Sharing}

In spectrum sharing, CR provides spectrum scheduling to overcome difficulties in open spectrum utilization. An optimal spectrum allocation technique is required among various Cognitive Radio users [2].

Cognitive radio was first introduced by Joseph. Mitola in 1999. J. Mitola introduced Radio Knowledge Representation Language (RKRL) which describes knowledge of radio environment and helps software define radio (SDR) in performing spectrum sensing [3]. Simon Haykin gave the brief over view of cognitive radio and describes cognitive radio as new technology in the field of wireless communication. Author focused on the uses of white space by using dynamic spectrum assignment and interference temperature to reduce interference in radio environment between transmitter and receiver by controlling the transmitter power [4].

Teng Joon Lim, Rui Zhang, Ying Chang Liang and Yonghong Zeng used GLRT principle to the problem of spectrum sensing in a CR network. Under assumptions of primary user's signal is present, they introduced eigenvaluebased algorithms which performs better than the conventional energy detector [5].The performance of the ED with estimated noise power (ENP) was analyzed by Andrea Mariani, Andrea Giorgetti, and Marco Chiani, they addressed the threshold design and gave the conditions for the existence of the SNR wall. they proved that noise estimation can avoid the SNR wall if the estimate is consistent with the observation interval[6]. Nguyen, Huan Cong, Carvalho, Elisabeth De,Prasad, Ramjee proposed blind spectrum sensing scheme in cognitive radio device which is equipped with multiple antennas. frequency domain spectrum sensing method applicable to frequency selective channels and receivers equipped with two antennas. SFCPS performs better than other correlation-based techniques [7]. Another spectrum sensing algorithms for cognitive radio networks was proposed by Tadilo Endeshaw Bogale, and Luc Vandendorpe. By assuming known transmitter pulse shaping filter, synchronous and asynchronous receiver scenarios were considered. In proposed algorithm a combiner vector is introduced, an over-sampled signal which has total duration equal to the symbol period is combined linearly. The Signalto-Noise ratio (SNR) minimization and maximization problems are formulated as Rayleigh quotient optimization 
problems for this combined signal. SNR minimization and maximization problems are formulated as Rayleigh quotient optimization problems for combined signal [8]. Iker Sobron, Paulo S.R. Diniz, Wallace A. Martins and Manuel Velez presented a new adaptive algorithm for spectrum sensing applications in cognitive-radio networks. The adaptive algorithm employs preprocessed information based on the energy estimates and noise variances coming from different neighbors in such a way that the deflection coefficient of the new test statistic is improved compared with that achieved in conventional ED techniques, where energy estimates are used directly. This key feature turned out to be very effective in the detection of white spaces in the radio spectrum [9]. Among all the spectrum sensing schemes energy detection (ED) is commonly used due to its low complexity. But for low signal to noise ratio (SNR) region the performance of ED scheme is poor. To enhance the sensing performance of ED scheme for low SNR region, an adaptive threshold was considered by Manish Gupta, Gaurav Verma and Rahul Kumar Dubey in . This adaptive threshold is a function of fixed threshold and SNR of primary user (PU) signal received at CR[10]. The improved cuckoo search algorithm to promote the capability of spectrum sensing in satellite cognitive systems was introduced by Wanmai Yuan, Mingchuan Yang, Qing Guo, Xinyu Wang, Xibao Feng . They consider the application scenario of satellite network, The sharable frequency bands are divided into a number of subchannels, and cognitive users should find unoccupied channels to access[11]. Md. Mehedi Hasan, Md. Mirajul Islam, Md. Imran Hussain, Sk. Mizanur Rahman proposed adaptive threshold detection model of energy detection based spectrum sensing. Through maximized the probability of detection with respect to a false alarm rate constraint in order to find the detector thresholds for each stage[12].

The structure of the paper is as follows. In Section II, system description is introduced, and the decision criterion to find unoccupied channels is given. In Section III, ACS algorithm is proposed. In Section IV, the simulation results for performance evaluation is described. Finally in Section V, the conclusion is given.

\section{SYSTEM DESCRIPTION}

The basic block diagram of proposed system is given below. To improve the detection speed of the unoccupied channels, Total wide frequency spectrum bands are divided into numbers of narrow band subchannels, then unoccupied channels are find out by Advanced Cuckoo Search(ACS). If any channel is found unoccupied then it can be assigned to secondary user.

$$
\text { RF Signal }
$$



Fig.1. Dynamic spectrum sensing with ICS

For spectrum sensing, Energy Detection(ED) technique is used. there are two hypothesis:
$\mathrm{H} 0$ : primary user signal is not present, only noise/interference is present.

$\mathrm{H} 1$ : primary user signal is present with interference

$$
\mathrm{E}(\mathrm{t})= \begin{cases}n(t) & H 0 \\ s(t)+n(t) & H 1\end{cases}
$$

In above equation $\mathrm{E}(\mathrm{t})$ is received signal , $\mathrm{s}(\mathrm{t})$ is the transmitted signal of the primary user, $n(t)$ is the additive white Gaussian noise. In energy detection(ED) the energy of received signal is measured. this measured energy is compared with a threshold value $\mathrm{E}_{\mathrm{th}}$.

-if E>Eth ,measured energy is greater than threshold value then primary user is present. Channel is occupied and that channel can not be allocated to secondary user.

-if $\mathrm{E}<\mathrm{Eth}$,measured energy is smaller than threshold value then primary user is absent. Channel is unoccupied and that channel can be used to secondary user.

\section{Spectrum Sensing by Advanced Cuckoo SeArch}

Cuckoo Search (CS) is a new search algorithm which has been developed by Yang and Deb in 2009. CS has been used in many fields of optimization with high efficiency.

Levy flights is a special feature of the Cuckoo Search algorithm for searching. Levy flight process is a random walk, which shows random searching pattern. Cuckoo bird lays eggs in the host nests (another bird's nest), if the host birds find the unknown eggs then they will discard the egg or abandon the nest. For surviving, Cuckoo eggs imitate the eggs of host birds. Three idealized rules used by the CS models are given below:

- Each cuckoo lays one egg, which is kept in a random host nest.

- The best eggs, or best solutions, will exist for next iteration.

- The number of host nests is fixed, and there is a probability $(0,1) \mathrm{Pa}$ that an unknown egg can be searched. Under this situation, the host birds can either discard the egg or leave the nest..

For Spectrum sensing by using Cuckoo search algorithm, $\mathrm{N}$ channels are generated and randomly a channel is selected by levy flight. Threshold for measured energy and time is fixed.so the characteristics of detected signal is compared with these thresholds and if signal strength is grater then threshold value then primary user is detected and if signal strength is less then threshold value then primary user is not present and that channel can be used by secondary user.

According to the research from Federal Communication Commission (FCC), The problem of lack of spectrum is mostly due to the inefficient utilization of the spectrum rather than the lack of the frequency resources. Improved Cuckoo search (ICS) is used in the optimization of capturing the unoccupied channels to avoid redundant search. Improved cuckoo search algorithm is capable to adjust dynamically the step size and switching/discovery probability to avoid repetitive search. ICS increases the convergence rate. The step size determines the scale of the search patterns which reduces the chances of search process to fall into the local 
Asian Journal of Convergence in Technology ISSN NO: 2350-1146 I.F-5.11

optima. The step size adjusted with the number of iterations. To maximize the detection speed of capturing the unoccupied channels, wide frequency bands are divided into numbers of narrowband subchannels, and CR operation just need to scan the subchannels, if any subchannel is not used by PUs then SUs can switch to the unoccupied channels by dynamic frequency hopping. In ICS Algorithm energy threshold is fixed but time threshold is adaptive and it is set according to previous signal information. [11].

We propose Advanced Cuckoo Search method which is more efficient for spectrum utilization as compared to CS and ICS. ACS algorithm is used to search the unoccupied subchannels. If any subchannel is unoccupied or primary user is not present then this subchannel can be allocated to secondary user. It improves the rate of capturing of subcannels and sensing time is also reduced. In advanced cuckoo search method thresholds for time and energy both are variable. For Spectrum Sensing, previous signals are analyzed and according to that information of previous signals, the value of thresholds for energy and time is evaluated which are adaptive thresholds. By using firm thresholding, adaptive thresholds can be evaluated.

For non-periodic signals we evaluate energy of those signals and for periodic signals, power calculation is done because energy of periodic signals is infinite. So in the case of periodic signals ACS gives more efficient results.

In ACS ,the process of searching unoccupied channel can be summarized as follows:

1. Generate $\mathrm{N}$ numbers of subchannels as host nest.

2. Choose a subchannel by levy flight. Evaluate its quality/fitness $\mathrm{F}$.

3.Compare quality/fitness with threshold $\mathrm{F}$.

4. if F>Threshold, Fitness/Quality is greater than Threshold value then channel is occupied by primary user and cannot be allocated to secondary user and if $\mathrm{F}<$ Threshold, Fitness/Quality is less then Threshold value then channel is unoccupied by primary user and it can be allocated to secondary user(SU).

Here Threshold keyword is used for both energy threshold and time threshold. and both thresholds are adaptive. It will first measure the energy of received signal and then compare this energy with energy threshold. if this energy is greater than energy threshold value then it is considerd that subchannel is occupied by primary user and can not be allocated to secondary user. and if measured energy is less then energy threshold then subchannel is considered unoccupied, now time duration of subchannel to remain unoccupied is compared with time threshold and if it is greater than time threshold only then this subchannel wiil be allocated to secondary user.

The whole process of Advanced Cuckoo Search can be understood by following flowchart.
Volume V Issue I

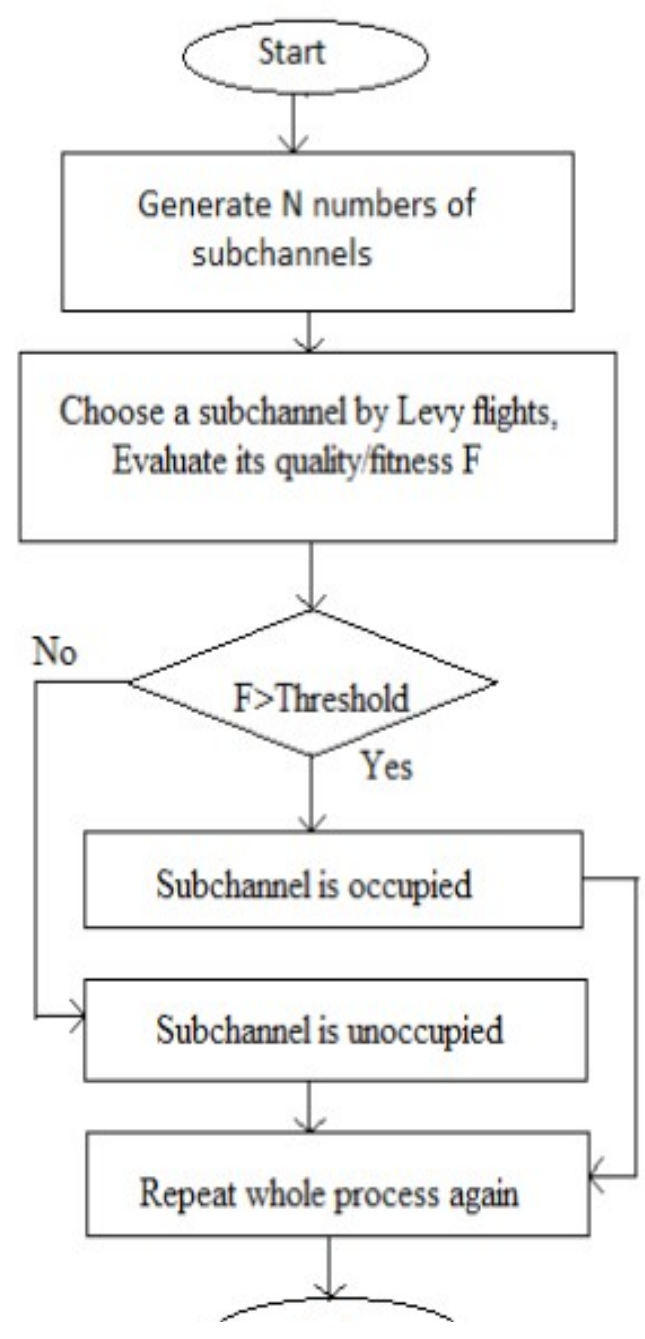

Fig.2.Flow chart of ACS

ACS provides frequency efficient spectrum utilization. Simple Energy Detection Technique is not efficient for periodic signals but ACS performs better because in ACS power is calculated for periodic signals because energy of periodic signals are infinite. It gives more efficient results for spectrum sensing and rate of capturing of subchannels as compared to CS and ICS .

\section{Simulation AND Results}

The validity of Advanced Cuckoo Search is verified by Simulation, which has been performed on matlab software. N numbers of subchannels are generated $(\mathrm{N}=32,64,128,256,512)$. BPSK modulation has been performed for signal transmission. By using ICS and ACS rate of capturing of subchannels is evaluated. Signal to noise (SNR) value is considered $-23 \mathrm{db}$. The Performance analysis is carried out on the basis of these plots-

\section{A. Improved Cuckoo Search}

In Fig.3 graph shows the performance of Improved Cuckoo Search(ICS). Graph is plotted between numbers of subchannels and Rate of Capturing of those subchannels. The rate of capturing of subchannels grows continually as the number of subchannels is added. 


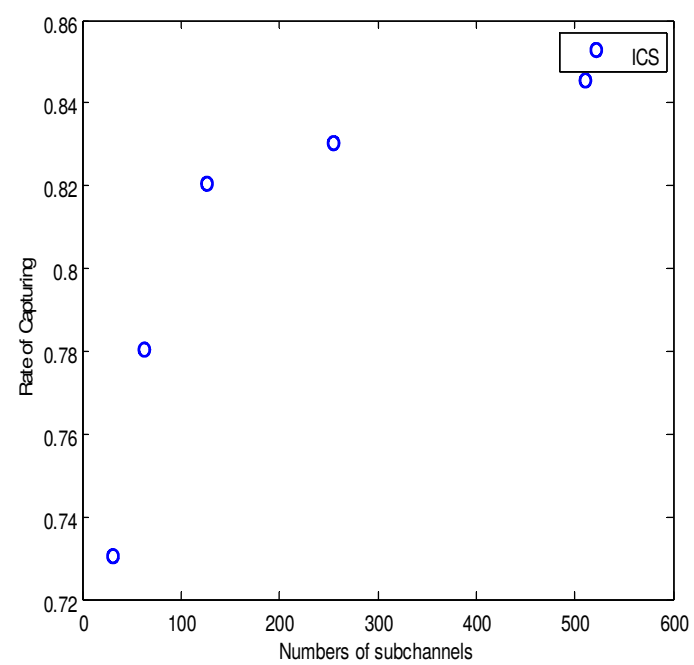

Fig.3. Numbers of subchannels v/s Rate of Capturing

\section{B. Advanced cuckoo search.}

In Fig.4 graph shows the performance of ACS in which rate of capturing of subchannels is higher than that of ICS.



Fig.4. Numbers of subchannels v/s Rate of Capturing

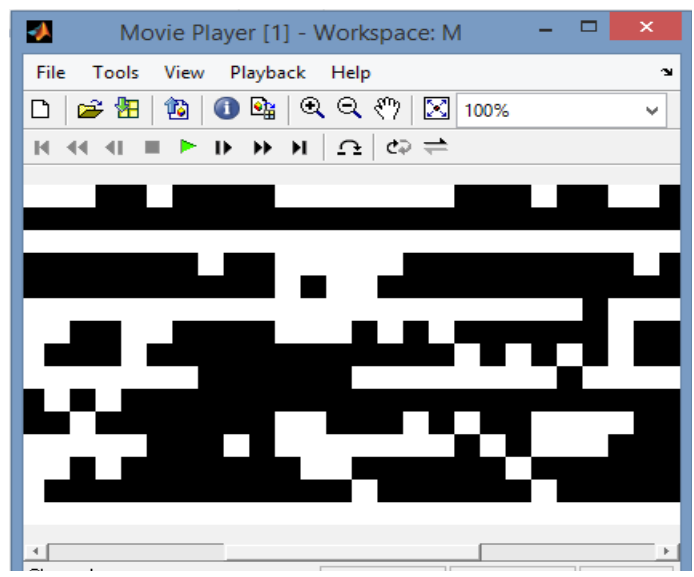

Fig.5.Frequency spectrum view
In Fig.5.white spaces shows the spectrum holes. Which can be allocated to secondary users. and black spaces shows the presence of primary user(PU), which can not be allocated to secondary users.

In Fig.6, graph shows Comparison between ICS and ACS

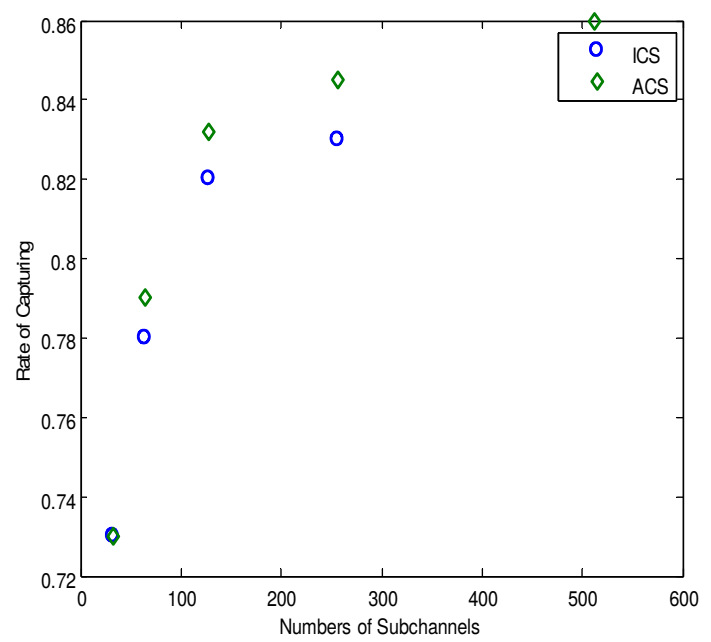

Fig.6. comparison between ICS and ACS

From the above Fig.6, it is clear that ACS gives better results for rate of capturing of subchannels as compared to ICS.

\section{CONCLUSION}

The radio frequency spectrum is the most precious natural resource for wireless communication. Observations on spectrum utilization reveal that several licensed bands are not used and remains unused for large time duration. Cognitive Radio (CR) is a favorable technology to enhance the efficiency of spectrum utilization.

The application scenario of spectrum sensing is performed in $\mathrm{Ku}$ band. The available frequency bands are divided into a number of subchannels. The CR sensing technique measures the quality/fitness of the subchannels to find unoccupied channels. These unoccupied channels can be used by secondary user for data transmission. Most importantly, an Advanced Cuckoo Search (ACS) algorithm is designed to improve the rate of capturing of subchannels and to give efficient utilization of frequency spectrum. Simulation has been done to show the performance of ACS and results of simulation show that ACS algorithm is more efficient as compared to other methods..

\section{REFERENCES}

[1] Niranjan Muchandi , Dr. Rajashri Khanai , "Cognitive Radio Spectrum Sensing : A Survey", International Conference on Electrical, Electronics, and Optimization Techniques (ICEEOT) 2016.

[2] Ahmed M. Jasim- MIEEE, Haidar N. Al-Anbagi “ AComprehensive Study of Spectrum Sensing Techniques in Cognitive Radio Networks". 2017 International Conference on Current Research in Computer Science and Information Technology (ICCIT), Slemani Iraq. 


\section{Asian Journal of Convergence in Technology ISSN NO: 2350-1146 I.F-5.11}

[3] Joseph Mitola, "Cognitive Radio: An Integrated Agent Architecture for Software Defined Radio," IEEE Personal Commuicat ions, pp. 13$18,1999$.

[4] S. Haykin, "Cognitive radio: Brain empowered wireless communications", IEEE Journal on Selected Areas in Communications, vol. 23 , February 2005.

[5] Teng Joon Lim, Rui Zhang, Ying Chang Liang and Yonghong Zeng, "GLRT-Based Spectrum Sensing for Cognitive Radio" unpublished.

[6] Andrea Mariani, Student Member, IEEE, Andrea Giorgetti, Member, IEEE, and Marco Chiani, Fellow, IEEE, "Effects of Noise Power Estimation on Energy Detection for Cognitive Radio Applications" IEEE Transactions on Communications, vol. 59, December 2011.

[7] Huan Cong Nguyen, Elisabeth de Carvalho and Ramjee Prasad, "Spectrum Sensing for Cognitive Radio Based on Multiple Antennas" , 2012 IEEE 75th Vehicular Technology Conference (VTC Spring).

[8] Tadilo Endeshaw Bogale, Student Member, IEEE, and Luc Vandendorpe, Fellow, IEEE, "Max-Min SNR Signal Energy Based Spectrum Sensing Algorithms for Cognitive Radio Networks with Noise Variance Uncertainty" IEEE Transactions on Wireless Communication, vol. 13, January 2014.

[9] Iker Sobron, Member, IEEE, Paulo S.R. Diniz, Fellow, IEEE, Wallace A. Martins, Member, IEEE, and Manuel Velez, Member, IEEE, "Energy detection technique for adaptive spectrum sensing" IEEE Transactions on Communications, in press.

\section{Volume V Issue I}

[10] Manish Gupta, Gaurav Verma and Rahul Kumar Dubey, "Cooperative Spectrum Sensing for Cognitive Radio Based on Adaptive Threshold" 2016 Second International Conference on Computational Intelligence \& Communication Technology.

[11] Wanmai Yuan, Mingchuan Yang, Qing Guo , Xinyu Wang, Xibao Feng, "improved Cuckoo Search Algorithm for Spectrum Sensing in Sparse Satellite Cognitive Systems" unpublished.

[12] Md. Mehedi Hasan, Md. Mirajul Islam, Md. Imran Hussain, Sk. Mizanur Rahman, "Improvement of Energy Detection Based Spectrum Sensing in Cognitive Radio Network Using Adaptive Threshold" IOSR Journal of Electronics and Communication Engineering (IOSR-JECE) e-ISSN: 2278-2834,p- ISSN: 22788735.vol. 13, Issue 2, ver. I (Mar. - Apr. 2018), PP 11-2.

[13] Ying-Chang Liang, , Kwang-Cheng Chen, , Geoffrey Ye Li, , and Petri Mähönen, "Cognitive Radio Networking and Communications: An Overview" IEEE Transactions on Vehicular Technology, vol. 60, September 2011.

[14] Sheetal Kokare, R.D.Kamble, "Spectrum Sensing Techniques in Cognitive Radio Cycle" International Journal of Engineering Trends and Technology (IJETT) - vol. 9, Mar 2014.

[15] Ekta Dua, Vikrant Gulati, "Enhancement in Spectrum Sensing Algorithms for Cognitive Radios" International Journal of Advanced Research in Electronics and Communication Engineering (IJARECE) vol. 5, August 2016. 